\title{
Memoria de actividades relacionadas con la innovación en la construcción: Documento de Idoneidad Técnica (DIT). 50 años de la UEAtc
}

\author{
Report on technical activities related to innovation in \\ construction: Spanish National Technical Approval (DIT). \\ 50 years of UEAtC
}

\section{A. Blázquez ${ }^{(*)}$}

\section{RESUMEN}

Anualmente la UEAtc (Union Européenne pour l'Agrément technique dans la construction / Unión Europea para la Idoneidad Técnica en Construcción), creada en 1960, y que por tanto celebrará su 50 aniversario el próximo año, reúne a los Directores de sus 18 Institutos Miembros, representantes, a su vez, de otros tantos países europeos, en la reunión anual denominada: Comisión de Coordinación. En esta Comisión, se discuten las situaciones nacionales y las interacciones entre las actividades de cada Instituto (País), para la evaluación de la innovación en la construcción y particularmente para la referida a los productos de construcción innovadores, en el ámbito del Documento de Idoneidad Técnica (DIT) (en otras lenguas Agrément, Avis Technique, etc.).

Como en muchas otras actividades que practicamos en nuestra vida, a veces, no prestamos suficiente atención a que éstas, se desarrollan en un contexto marcado por hechos históricos más o menos relevantes que se suceden paralelos a nuestra actividad profesional, a la que sin duda de una manera u otra afectan significativamente. Es por ello, que se realiza aquí, una reflexión con ánimo constructivo y optimista, un ejercicio simple subjetivo y con alguna dosis de humor, que lista, paralelamente a algunos de los hechos históricos anuales que han ido aconteciendo, aquellos otros, más técnicos, que tienen que ver con el DIT. Exposición que se finaliza con el Resumen Anual (2008), de actividades del DIT, presentado a la última Comisión de Coordinación de la UEAtc.

El mensaje es doble: por un lado no deberíamos olvidar la historia y lo que hemos conseguido, y por otro lado, nuestro trabajo no debería abstraerse en sí mismo, hasta el punto de perder de vista la relatividad de lo que hacemos.

074-5

Palabras clave: Documento de Idoneidad Técnica, DIT, UEAtc, Innovación.

\section{SUMMARY}

Every year the UEAtc (Union Européenne pour l'Agrément technique dans la construction / European Union of Agrément), which was founded in 1960 and will therefore be celebrating its $50^{\text {th }}$ anniversary next year, convenes the directors of its member institutes, each representing its home country, to a meeting of the Co-ordination Commission. This Commission discusses national circumstances and the interaction among the activities conducted by the respective Institutes (countries) in connection with the assessment of construction industry innovations, particularly innovative products, procedure called in Spanish, Documento de Idoneidad Técnica (in other languages: Agrément, Avis technique and so on).

As it is for other of life's activities, so it is with technical approvals, that at times we pay little heed to the fact that they take place in a context marked by relevant historic events occurring during our careers, which are significantly affected by these situations in one way or another. This is what underlies the present constructive and optimistic reflection, a simple and subjective exercise with some sense of humour that lists some of the yearly highlights coetaneous with other more technical events related to technical approvals. This discussion concludes with the annual summary of activities in 2008 submitted to the March 2009 meeting of the UEAtc's Co-ordination Commission.

The message conveyed is that while on the one hand we must not ignore history and what we have achieved to date, on the other our efforts should not become so self-involved as to lose sight of the relativity of our endeavours.

Keywords: Technical Approval, DIT, UEAtc, Innovation.

* Instituto de Ciencias de la Construcción Eduardo Torroja. Madrid (España)

Persona de contacto/corresponding author: blazquez@ietcc.csic.es

Fecha de recepción: 15-10-09 Fecha de aceptación: 10-11-09 


\section{INTRODUCCIÓN}

El año 2008 se recordará por la crisis financiera mundial que afectó de manera dramática a todos los sectores de producción, pero en España sobretodo, al sector de la construcción.

La crisis fue, para casi todos, imprevisible y sus efectos sobre la economía y la construcción española, están aún por determinar. Desde el punto de vista de los productos de construcción y especialmente de los productos innovadores de construcción, el efecto de la crisis ha sido bastante desigual. Por un lado es evidente que existen muchas menos oportunidades (obras en construcción) para el empleo de productos, lo que limita el interés por innovar, pero por otro lado, muchas empresas desean aprovechar, en tanto la situación no se prolongue más allá de lo "máximo permisible", la oportunidad de "inventar", no sólo como refugio en tiempos de inactividad, también porque es el mejor soporte en la lucha que supone la competitividad. El interés por la innovación es al fin y al cabo, una característica de las sociedades más desarrolladas.

Las actividades relacionadas con la innovación en construcción y específicamente las de aquellas que tienen por objetivo evaluar de forma previa a su uso, la idoneidad técnica de los productos no tradicionales o innovadores, están ligadas a la actividad de la Organización Europea: UEAtc (Union Europénne pour l'Agrément technique dans la construction). Una Unión que agrupa a los Institutos de 18 países europeos (el IETcc fue cofundador), de los 27 de la UE (2 países pertenecen a la
EFTA (European Free Trade Association)), reconocidos por sus respectivos Estados para la concesión del Documento de Idoneidad Técnica (DIT), en otras lenguas: Agrément, Avis Technique, Technical Approval, Technical Appraisal, Documento de homologaçao, Zulassung, Agrément Tecnico, etc.

La historia de la UEAtc se remonta a 1959 y su situación actual es el resultado de la sucesión de acontecimientos acaecidos durante todos estos años. Acontecimientos técnicos y también históricos, como no podía ser de otra forma. Por eso, recordar paralelamente la historia para ambos aspectos, aunque sea de forma breve, puede ser un buen ejercicio que nos permita comprender mejor lo que hemos hecho y dónde nos encontramos, y sin duda, el mejor camino para decidir dónde vamos y qué queremos del futuro. Un ejercicio simple subjetivo y con alguna dosis de humor, que termina con el resumen del Informe Anual del 2008 sobre las actividades del DIT en España, presentado a la reunión 55 de la Comisión de Coordinación del 2009.

Actualmente, la UEAtc, condicionada tanto por los numerosos cambios que se están produciendo en el ámbito europeo, -por ejemplo, la revisión de la Directiva de Productos de Construcción-, como por la natural evolución de los intereses de sus Miembros, está revisando de forma sustancial su estatus de Organización. Su futuro, después de 50 años de funcionamiento, está, en este momento, siendo hondamente discutido y aunque la UEAtc tiene sólidas raíces, el viento del cambio sopla fuerte y cualquier evolución es posible.

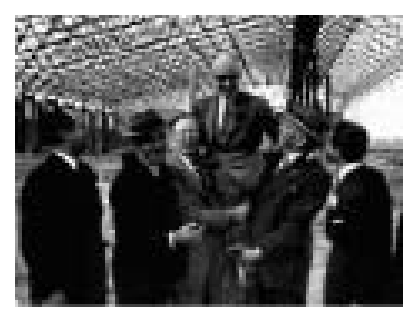

Profesor Eduardo Torroja.
(...) Desde la creación de la UEAtc hasta la actualidad. Un paseo por la historia. ¿Qué pasos fundamentales se han ido dando para el desarrollo de la evaluación de la innovación en construcción, y qué ocurría mientras en el mundo?:

1959: El año que triunfa la revolución cubana, Franco inaugura el monumento del Valle de los caídos. Se forma la ETA. Se finaliza el Museo Guggenheim en Nueva York del arquitecto Frank Lloyd Wright. René Goscinny y Alberto Uderzo publican el primer libro de Asterix. El Real Madrid gana su cuarta Copa de Europa consecutiva (conseguirá otras cinco más (por el momento)). (...)

Eduardo Torroja, fundador del IETCC, junto con Gerard Blachère del CSTB, iniciaron una colaboración destinada a analizar, la creación de una Organización para el desarrollo y reconocimiento de procedimientos que permitieran el estudio de los materiales y sistemas constructivos no tradicionales o innovadores, a fin de facilitar su incorporación a las obras de construcción.

Crearon las bases de la UEAtc y del DIT español (Documento de Idoneidad Técnica), basado en el "Avis Technique" francés, proponiendo un "nuevo enfoque" para el estudio de los productos y sistemas constructivos; un enfoque basado en prestaciones, que sería la base del trabajo pasado y presente de los DIT, como también el elegido por nuestra Ley de Ordenación de la Edificación de 1999 y su desarrollo en el Código Técnico de la Edificación, aprobado en el año 2006 (cuarenta y siete años después).

Hay que tener en cuenta que en la década de los 50, España era un país primordialmente agrícola, y que el papel desarrollado por el entonces Ilamado "Instituto Técnico de la Construcción y del Cemento" (itcc) -antes llamado Instituto Técnico de la Construc- 
ción (itc)- dirigido por Torroja, como Centro Tecnológico y de Investigación, fue único y precursor, tanto en nuestro ámbito nacional, como en la apertura española al exterior.

1960: El año que se crea la OPEP (Organización de Países Exportadores de Petróleo) y también la EFTA (Asociación Europea de Libre Comercio). J.F. Kennedy gana las elecciones presidenciales de Estados Unidos. Se comercializan las primeras píldoras anticonceptivas. (...)

En Junio, se reúnen en París, representantes del CSTB (Francia), CORI Group (Italia), RATIOBOUW Foundation (Holanda), LNEC (Portugal), INL (Bélgica) e itcc (España), que preparan el primer borrador de estatuto para una asociación, cuyo fin será la evaluación de la idoneidad técnica de los productos innovadores de construcción. El 10 de octubre de ese mismo año, los 6 Institutos reunidos en Madrid crean formalmente la UEAtc, con el profesor Eduardo Torroja representando al itcc. Paulin Roger del CSTB francés, sería elegido Secretario General. Lo será hasta su jubilación en 1982.

1961: El año que muere ( 15 de junio) el Profesor Eduardo Torroja. El centro fundado por él, pasa a llamarse en su memoria: "Instituto Eduardo Torroja de la Construcción y del Cemento (ietcc)". Se levanta el muro de Berlín. (...)

La UEAtc establece los primeros contactos con Institutos de Alemania, Reino Unido, Grecia y Luxemburgo.

El nuevo Director del IETcC, Jaime Nadal continúa la labor iniciada por Eduardo Torroja, apoyando sin reservas el desarrollo del DIT. Lo hará hasta el año 1968, en el que le sustituye Fernando Casinello. El ietcc inicia ese año, los trabajos para la concesión del primer DIT.

1962: El año que España solicita por primera vez su ingreso en el Mercado Común Europeo. EI Papa Juan XXIII excomulga a Fidel Castro. Comienza el bloqueo de EEUU a Cuba. Se forman los Rolling Stones. (...)

La UEAtc se amplía con el Istituto per il Certificato d'Idoneitá tecnica (ICITE), de Italia.

El 20 de septiembre, se concede el primer DIT español al Sistema Fiorio. Un DIT resultado de la convalidación del Avis Technique $\mathrm{n}^{\circ}$ 1466 sobre prefabricación pesada, concedido por el Instituto francés: Centre Scientifique et Technique du Bâtiment (CSTB).

Antonio Ruiz Duerto (1933-1999), Dr. Arquitecto, Coordinador del DIT hasta

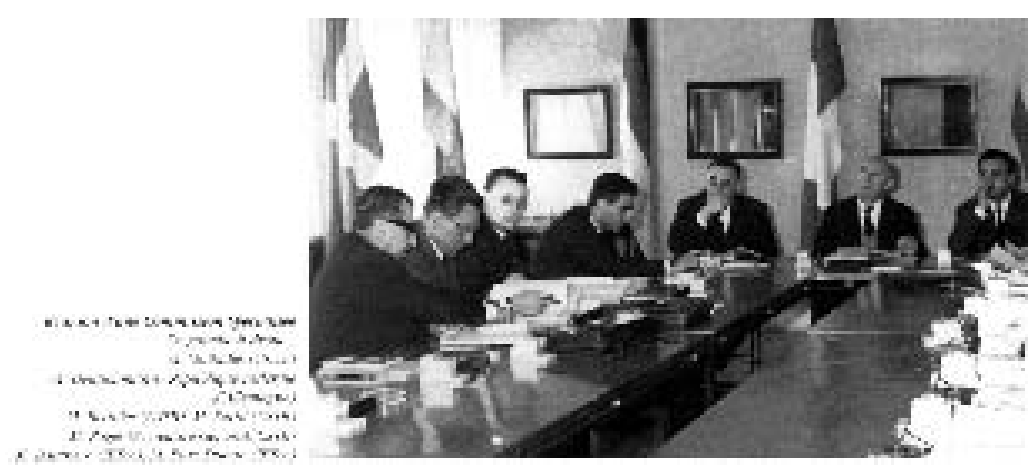

1990, actuó como Ponente de aquella primera convalidación, en un trabajo que fue significativamente reconocido posteriormente por la UEAtc, en su reunión anual del año 2000 (40 aniversario) celebrada en el IETcC, considerando a nuestro maestro Ruiz Duerto como el "primer pionero" de la Organización.

La revista Informes de la Construcción, que había comenzado a editarse por el Torroja en 1948, comienza a "encartar" los Documentos de Idoneidad Técnica y a difundir la publicación de las Directivas UEAtc, publicadas en forma de Monografías independientes, traducidas por los técnicos del IETcc y anexadas como "Cuadernos de Informes". La colaboración entre la revista y el DIT se ha mantenido desde entonces.

Con Antonio Ruiz Duerto, estarán siempre en deuda la UEAtc, el Torroja y esta revista, Informes, a la que ayudó a crecer de manera entusiasta.

1963: El año de la manifestación por los derechos civiles en Washington D.C, en la que Martin Luther King pronuncia su célebre discurso: "I have a dream" (Tengo un sueño). Asesinan (22 de noviembre) a J.F. Kennedy en Dallas. Los Beatles editan su primer disco: "Please please me" y Bob Dylan publica "Blowing in the wind". (...)

La UEAtc se amplía con la entrada del "Central Public Works Laboratory" de Grecia, que desafortunadamente se retira en 1966. Austria entra como observador, condición que mantendrá hasta el fin de la década.

Se publica el Decreto 26 de diciembre de 1963 de Presidencia del Gobierno, que faculta al entonces denominado Instituto Eduardo Torroja de la Construcción y del Cemento, para conceder el Documento de Idoneidad Técnica. El Decreto, en su párrafo segundo indicaba:

"No regular la utilización de tales productos (nuevos materiales, elementos

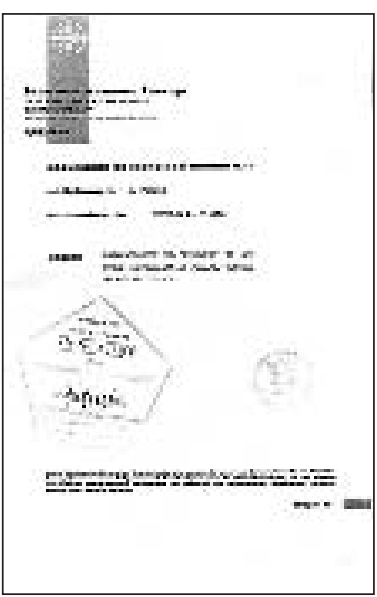

Portada DIT n 1 (1962)

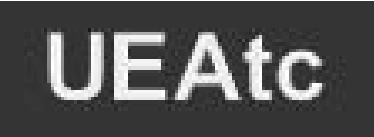

Símbolo UEAtc.

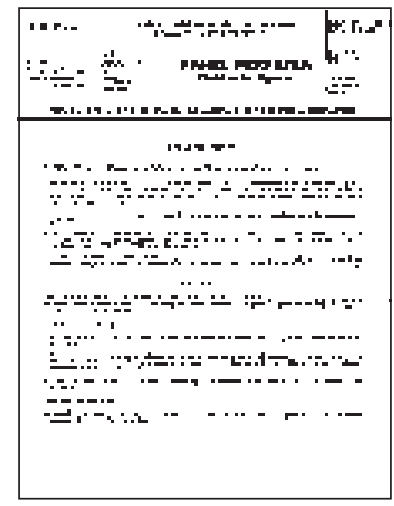

2a portada DIT (n² 2 a nº 99). 
y procedimientos de construcción) puede entrañar riesgos importantes y su desestimación podría ser causa de retraso nacional en construcción, tanto de edificios como de obras públicas, por no tomar en consideración las nuevas aportaciones que, cada día más numerosas, surgen en todo el mundo en este sector de actividad"

1964: El año que Guinea Ecuatorial se independiza de España. La empresa IBM presenta su primer modelo de ordenador: Serie 360. Nelson Mandela pronuncia su famoso discurso: "Estoy listo para morir". Se publica la primera tira de Mafalda, en Argentina. España gana su primer campeonato de Europa de fútbol al vencer a Rusia por 2 a 1 (Goles de Pereda y de Marcelino). (...)

El "Bundesministerium für Wohnungswesen, Städtebau und Rumordung" de Alemania, entra como observador en la UEAtc.

El Instituto Torroja incorpora su primer ordenador o cerebro electrónico, el NCR-Elliot 803 B, uno de los más potentes del país.

La UEAtc publica sus primeras Directivas Comunes: Fachadas ligeras (Light Claddings) y Tabiques de yeso (Gypsum Plaster Partitions). Inicialmente, el término empleado era "Jurisprudencia", sustituyéndose en abril de ese año por el de "Directiva Común", y posteriormente por el de "Guía Técnica". Hoy se llaman "Informes Técnicos".

1967: El año de la guerra de los seis días que enfrentó a Israel con Egipto, Siria y Jordania. Nieva en la ciudad de Méjico. EEUU, URSS y Reino Unido firman el Tratado del Espacio Exterior. Matan al Che Guevara en Bolivia. (...)

El "British Board of Agrément" (BBA) del Reino Unido, entra como observador en la UEAtc.

Se traduce al español por el Dr. Arquitecto Buenaventura Bassegoda, el libro de Gerard Blachére, Director del CSTB, titulado: "Savoir Batir: Habitabilité, durabilité, economie des bâtiments", (Saber construir: Habitabilidad, durabilidad, economía de los edificios), escrito un año antes. Un libro considerado largo tiempo como la "biblia" de la evaluación prestacional de productos de construcción.

1968: El año (mayo) de la revolución universitaria en Francia. Asesinan a Martin Luther King. España gana el festival de eurovisión con Massiel y el tema la, la ,la. Primer asesinato de la banda terrorista ETA: el guardia civil, José Ángel Pardines. Rusia invade Checoslovaquia. Richard Nixon es elegido presidente de EEUU. (...)
La UEAtc se amplía con la entrada definitiva del BAM de Alemania y el BBA del Reino Unido (ya son ocho miembros). Austria sigue como observador. El Torroja llega ese año hasta la concesión de DIT no 19 y además a la confirmación C-9, que hasta el año 1969 se numeraban de forma separada.

1969: El año que (según nos contaron) el Apolo 11 llegó a la luna y Neil A. Armstrong la pisó por primera vez. (...)

El IETcc concede ese año 5 DIT y llega al $\mathrm{n}^{\circ} 25$. Las renovaciones (una) se numeraban hasta entonces, con un número nuevo. La UEAtc publica su Directriz UEAtc para la apreciación de Puertas y el IETcc publica la traducción de la Monografía (7) sobre Directrices Comunes a base de Paneles Prefabricados Pesados, realizada por la UEAtc en 1966.

1973: El año de la crisis del petróleo, la OPEP duplica sus precios. Robo del banco Kreditbanken en Estocolmo, que daría lugar al conocido "síndrome de Estocolmo". ETA asesina a Carrero Blanco. (...)

El año en el que la Comisión Europea crea el "mercado interno" y se inician los primeros contactos entre Miembros de la misma y la Comisión de Coordinación de la UEAtc.

El Torroja concede 11 DIT e inicia la redacción como Rapporteur (Ponente) de la primera Directriz UEAtc: Assessment of Windows (Evaluación de ventanas), que se finalizará al año siguiente.

1975: El año que Bill Gates y Paul Allen fundan Microsoft. Bobby Fisher renuncia a defender su título frente a Anatoly Karpov. Muere Franco. Juan Carlos de Borbón es proclamado Rey de España. Según Wikipedia, la población mundial supera los 4.000 millones de personas. (...)

Se revisan por primera vez los Estatutos de la UEAtc y se crea la Comisión de Confirmación, para debatir las tramitaciones de las confirmaciones/convalidaciones de DIT entre Institutos.

1976: El año del primer vuelo comercial del Concorde. Se funda la Apple Computer Company. Adolfo Suárez es nombrado presidente del gobierno español. Se celebra el XXVII Congreso del PSOE después de 32 años en el exilio. Primer concierto de los Rolling Stones en España, en Barcelona. (En 1982 lo harán en Madrid). (...)

Se revisa por primera vez el Reglamento Interior de la UEAtc. El Instituto FWBP de Austria se convierte por fin en Miembro 
de la UEAtc, aunque se retirará en 1989. Al mismo tiempo, el IIRS de Irlanda y el SBI de Dinamarca son aceptados como observadores.

1977: El año que asesinan a cinco abogados laboralistas en Madrid: "La matanza de Atocha". Se legalizan PSOE y PCE. Se inaugura el Centro Pompidou en París, también conocido como "Beaubourg", del arquitecto Renzo Piano, todavía uno de los más modernos museos jamás construidos. Fallece Elvis Presley. (...)

El Gobierno Español aprueba el marco unificado para la normativa de la edificación, compuesto por: Normas Básicas (NBE), Norma Tecnológicas (NTE), Soluciones homologadas de la edificación (sin desarrollo) que completarían a los Documentos de Idoneidad Técnica.

El IETcc incorpora en el DIT $n^{\circ} 100$, el nuevo logotipo del DIT, fruto de un concurso interno de diseño. Logotipo que se conserva hasta la fecha.

1978: El año que en Nicaragua los sandinistas anuncian la guerra civil. China levanta la prohibición sobre las obras de Aristóteles, Shakespeare y Dickens. España aprueba la Constitución Española. Se despenaliza el adulterio. (...)

La UEAtc publica sus primeras Reglas para el DIT: La R.01 sobre el contenido del DIT, la R.02 para el reconocimiento recíproco de los ensayos del DIT, la R.03 para la certificación de conformidad con el DIT y su reconocimiento recíproco. Todas ellas serán revisadas en 1984 .

1979: El año que vuelve a legalizarse la francmasonería en España. Primer lanzamiento con éxito de un cohete europeo, el Ariane 1 de la Agencia Espacial Europea. (...)

Se publica el primer boletín de la UEAtc con una tirada de 14.000 ejemplares, siendo su editor en jefe Aldo Franchi, director del ICITE Italiano. El boletín se publicó hasta el número 41, año 2000 y siempre en inglés, francés y alemán.

1980: El año que se crea la CNN. Se celebran los Juegos Olímpicos en Moscú con el boicoteo de EEUU y algunos de sus aliados. Iraq invade Irán. Ronald Reagan es elegido presidente de EEUU. Matan a John Lennon en Nueva York. (...)

Se firma el Convenio de Roma, para los ocho miembros de entonces de la CEE, que entró en vigor en 1991. El Instituto KOMO entra en la UEAtc, representando a Holanda, sustituyendo al BC. Ya son 10 miembros y un observador, el IIRS de Irlanda.

El director del Torroja desde 1971, Francisco Arredondo es sustituido por Fernando Aguirre, que lo será hasta 1983.

1983: El año de la nacionalización de RUMASA por el Gobierno Español de Felipe González. Aparece por primera vez en el mercado el CD. El Papa Juan Pablo II retira la condena a Galileo Galilei. Un avión de Iberia colisiona con otro de Aviaco en el aeropuerto de Madrid: Mueren 93 personas. Sólo diez días antes en el mismo aeropuerto un avión de Avianca se había estrellado: 181 muertos. España gana a Malta por 12 goles a 1. (...)

Sustituyendo a Paulin Rogier, Emile Farhi también del CSTB de Francia, es elegido Secretario de la UEAtc, cargo que ocuparía hasta 1998. El Instituto IIRS de Irlanda, es por fin acogido como miembro de la UEAtc (ya son 11 Miembros). El Torroja actúa como Ponente de la Directriz UEAtc Plastic Renderings (Revestimientos plásticos para fachadas).

Juan Murcia es nombrado director del IETCC.

1985: El año que España reabre la verja con Gibraltar. En Uruguay se restaura la democracia. Mijail Gorbachov se convierte en Presidente de la Unión Soviética. España firma (12 de junio) el tratado de adhesión a la Comunidad Económica Europea. Sergei Bubka supera con pértiga los 6 metros de altura. (...)

La CE prepara el "Libro Blanco", decidiendo adoptar una "nouvelle approche" para la armonización europea, vía referencia a las exigencias esenciales a los productos y a las obras.

El Torroja concede ese año 12 DIT, de los cuales 3 son renovaciones y 3 confirmaciones de otros DIT concedidos por Institutos de la UEAtc, que éste año cumple 25 años.

María del Carmen Andrade es nombrada directora del IETCC.

1986: El año que España entra en la Comunidad Económica Europea (hoy Unión Europea). En EEUU el transbordador Challenger estalla poco después del despegue. La OPEP baja el precio del petróleo. La CEE adopta la bandera europea. Catástrofe nuclear en Chernobil. (...)

La Asociación Española de Normalización (AENOR) es reconocida mediante Real

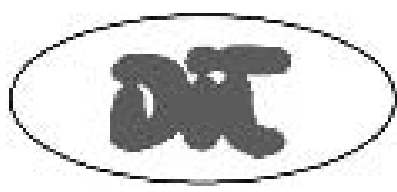

Logo DIT.

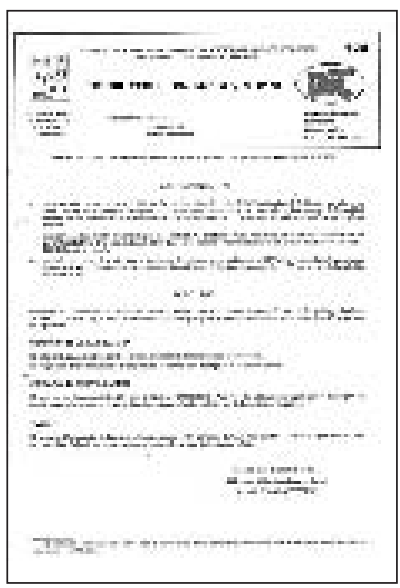

Portada DIT $n^{\circ} 100$ (incluye el logo por $1^{\text {a }}$ vez). 
Decreto 1614/1985, como Organismo de Normalización. Será también reconocida en 1995 como Organismo de Certificación (RD 2200/1995).

La UEAtc publica su Directriz $n^{\circ} 32$, para Colectores solares con circulación líquida; en la que debe reseñarse el Prólogo de F. Braun, General Director GM III. Internal Market and Industrial Affairs, de la CEE, que reconoce la participación en su redacción, de la CEE y particularmente del "General Managment XII Science, Research and Development. En dicho Prólogo, F. Braun considera a las Directrices UEAtc como base para el "European Technical Approval", en el borrador en curso de preparación de la Directiva de Productos de Construcción, reconocimiento que se había ya incluido en la Directriz UEAtc $\mathrm{n}^{\circ}$ 30: Insulating Glazing.

1987: El año que según la ONU había 5.000 millones de personas en el mundo. Reagan y Gorbachov firman en Washington un tratado de eliminación de armas nucleares. (...)

La CE modifica el Tratado de Roma adoptando el Acta Única. La UEAtc comienza el desarrollo, a solicitud de la DG III de la CE, de un prototipo de DIT Europeo (lo que será el DITE) para un producto de plástico destinado al revestimiento de suelos.

El Torroja actúa como Ponente de la Directriz especial UEAtc Impermeabilización de cubiertas en APP (Atactic Polipropilene).

1988: El año que Pinochet es derrotado por un plebiscito en Chile y George Bush (el padre) es elegido Presidente de EEUU. La ONU reconoce el Estado Palestino. Antonio Mingote toma posesión del sillón $r$ de la RAE. (...)

Tras un largo trabajo coordinado por la dirección y Unidad del DIT, del IETcC, se publica el Reglamento para la concesión del DIT, que aparece en nuestro BOE el 18/01/09. El Reglamento, que se propone para "facilitar el cumplimiento de los compromisos del IETCC con respecto a la UEAtC", reconoce al DIT como elemento que, "antes de la propia existencia de la CEE, ha permitido disminuir las barreras existentes entre los países con respecto a materiales y procedimientos no tradicionales".

El Reglamento, introduce la creación de una Comisión Técnica del DIT, integrada inicialmente por la Dirección General de Industrias Químicas de la Construcción del MINER, la Dirección General de Innovación Industrial y Tecnológica, del MINER, Dirección General para la Vivienda y la Arquitectura, del MOPU, la Secretaría
Técnica, del MOPU, la Confederación Nacional de la Construcción (CNC), la Asociación Española de Normalización y Certificación (AENOR) y el propio IETcC, cuya misión es la de velar por la adecuada concesión y homogeneidad de los DIT, así como proponer a la UEAtc, a través del IETcc, la redacción de nuevas Guías.

El Reglamento también establece, para asesorar al IETcC, la formación de Comisiones de Expertos para cada concesión de DIT. En la actualidad se invita a participar a Organismos e Instituciones tales como: Entidades de Control de Calidad, Asociaciones de Fabricantes, Colegios Profesionales (arquitectos, arquitectos técnicos, ingenieros), Constructoras, Centros de Investigación, laboratorios, AENOR, Organismos oficiales (Ministerios, Ayuntamientos), etc.

Se crea la Unión Española para la Idoneidad técnica de la construcción (UEltc) mediante Convenio específico entre la Dirección General de Vivienda y Arquitectura y el CSIC. Su objetivo era el de coordinar el trabajo del DIT en la UEAtc y definir las tareas de seguimiento de los DIT, las cuales serían -inicialmente- realizadas por la Subdirección General de Normativa Básica y Tecnológica. Sin embargo, la UEltc, salvo la asistencia conjunta a las reuniones de la UEAtc y algún trabajo específico en el campo de los impermeabilizantes, no se consolidó; aunque la colaboración entre el IETcc y el Ministerio de Obras Públicas y Urbanismo, hoy Ministerio de Vivienda, se mantiene en la actualidad.

Se desarrolla el Diagnóstico de Innovación Europeo (DIE), un "dossier técnico" de ayuda a las empresas e industriales que deseaban lanzar un nuevo producto. Una evaluación para establecer ventajas, riesgos y mejoras o modificaciones posibles para los productos que deseaban ser introducidos en otros países.

Inicialmente el DIE fue un acuerdo entre los institutos CSTB (Francia), CSTC (Bélgica), ICITE (Italia), LNEC (Portugal) y el IETcC (España). Un proyecto interesante que sin embargo no logró consolidarse.

Las Directrices Comunes de la UEAtc pasan a denominarse Guías Técnicas de la UEAtc (UEAtc Guides to Agrément). La UEAtc firma un acuerdo de colaboración con el Comité Europeo de Normalización (CEN).

Se publica, el 21 de diciembre, la Directiva de Productos de Construcción 89/106/CEE, la DPC; modificada posteriormente en 1993 y actualmente en curso de nueva revisión. 
Rafael Blázquez es nombrado director del Torroja.

1989: El año que entra en vigor el Protocolo de Montreal para proteger la capa de ozono. El primero de los 24 satélites que conforman el "Global Positioning System" (GPS) o Sistema de Posicionamiento Global, es puesto en órbita. Jomeini ofrece una recompensa de tres millones de dólares por la muerte del escritor Salman Rushdie. Se inaugura la pirámide del Louvre. Cae el muro de Berlín. (...)

El Instituto Torroja cambia su nombre a Instituto de Ciencias de la Construcción Eduardo Torroja.

El 27 de diciembre el MINER notifica de forma provisional al IETCC como Organismo español portavoz para la concesión del DITE (Approval Body) (único Organismo, hasta la notificación del ITEc de Barcelona, en el año 1993).

Se inicia la redacción por la CEE, de los Documentos Interpretativos, en desarrollo de los Requisitos Esenciales enunciados en el anexo I de la DPC.

La UEAtc acepta como observadores a los Institutos BOVERKET de Suecia, NBRI de Noruega y VTT de Finlandia. El Instituto SBK de Holanda, sustituye a KOMO.

La UEAtc en su Boletín especial de marzo publica el resultado del ejercicio piloto para un "European Technical Approval" para un producto de plástico destinado al revestimiento de suelos. Participaron los Institutos: BAM (Alemania), BBA (Reino Unido), ICITE (Italia) y el IETcc (España). Un documento, que no será finalmente desarrollado por la UEAtc ni la CEE, aunque se tendrán en cuenta sus aportaciones en la futura EOTA (European Organization for Technical Approvals) creada a la aprobación de la DPC.

La UEAtc prepara (será aprobada el año siguiente) la Regla Práctica $n^{\circ} \mathbf{1}$ relativa a la denominación comercial de los productos de construcción. (Publicada en el Boletín $\mathrm{n}^{\circ} 21$ ).

1990: En Sudáfrica Nelson Mandela es liberado después de 27 años de cárcel. La RDA y la RFA se unen en una sola Alemania. Hollywood concede el oscar honorario a Sofía Loren. (...)

Se reúnen en París, por primera vez, los miembros notificados por sus Estados Miembros para la concesión de los Documentos de Idoneidad Técnica Europeo (DITE), (ETA en inglés). Siguiendo las indicaciones del Anexo II de la DPC 89/106/CEE, que reque- ría la creación de una nueva Organización, formarán la EOTA, nombre que, por tanto, no aparece en la DPC.

La UEAtc incorpora como Miembros al BOVERKET, NBRI y VTT. (Ya son 14 Miembros).

En el IETCC, Vicente Mas Sarrió, que fue también director de esta revista Informes hasta el año 1981, sustituye como Responsable del DIT a Antonio Ruiz Duerto, que deja temporalmente el IETcC.

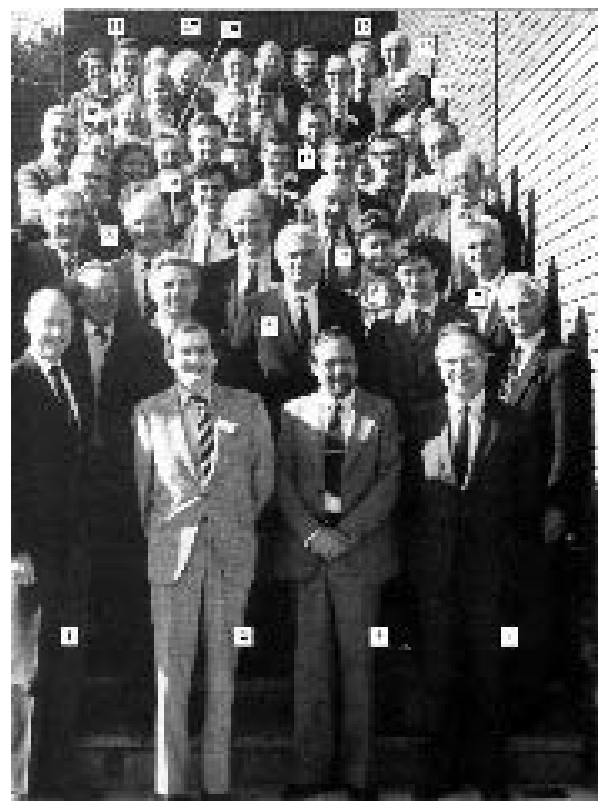

1991: El año que la CE expedienta a España por la excesiva suciedad de sus playas. Comienza la guerra del Golfo. Se crea el Instituto Cervantes. Deja de existir, como Estado, la Unión Soviética. (...)

El MINER notifica de nuevo al IETcc como Organismo español portavoz para la concesión del DITE. El Torroja concede ese año 21 DIT, de los cuales 4 son renovaciones y 5 confirmaciones de otros DIT concedidos por Institutos de la UEAtc.

Se finaliza la Guía UEAtc Complementaria para la evaluación de impermeabilizaciones de cubiertas fijadas mecánicamente, que será tenida en cuenta en su totalidad para la redacción de la Guía EOTA N ${ }^{\circ} 06$ Sistemas de impermeabilización de cubiertas con membranas fijadas mecánicamente, editada en el año 2000. Un proceso que se repetirá en muchas de las Guías elaboradas por EOTA.

1992: Quinto centenario del descubrimiento de América. Se firma el tratado de Maastricht, que asocia la CECA, CEE y CEA de la Comunidad europea. Barcelona organiza los Juegos Olímpicos y Sevilla la Exposición Universal. La
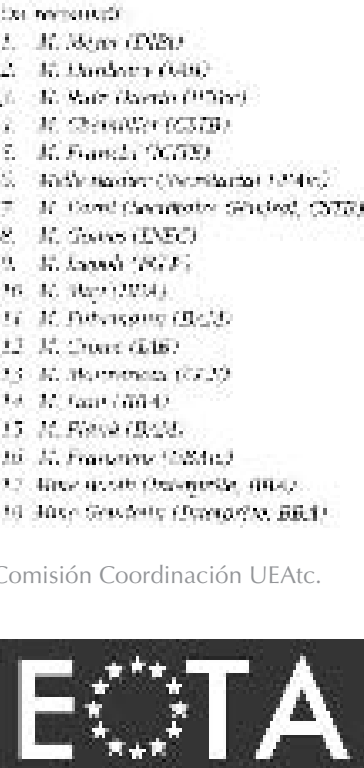

Logo EOTA 
Compañía AT \&T presenta el video teléfono. Se inaugura el AVE Madrid-Sevilla. Se detecta una importante disminución de la capa de ozono sobre el Ártico y los países del norte de Europa. Se forma la Unión Europea (UE), que junto con la EFTA acuerdan la creación del Espacio Económico Europeo (EEE): El mercado único. (...)

Se publica el Real Decreto 1630/1992 (modificado posteriormente por el RD 1398/1995) por el que se traspone (se establecen disposiciones necesarias para la aplicación) la DPC al Estado Español. El RD regula la notificación de los Organismos de Certificación, Inspección y Laboratorios de ensayo (Approved Bodies), así como los que denomina Organismos de Concesión del DITE (Approval Bodies). El RD reconoce al IETcc en su disposición adicional segunda: Organismos facultados para conceder el DITE, al considerar lo previsto en el Decreto 3652/1963 de 26 de diciembre. También en el Anexo IV establece las condiciones para la autorización de otros organismos de concesión del DITE.

Dicho RD establece, además, en su disposición final primera, la formación de la Comisión Interministerial para los Productos de Construcción (CIPC) como órgano de apoyo y coordinación para la aplicación del $\mathrm{RD}$, la cual reconoce como vocal miembro al IETcC.

Se plantea mediante borrador de Orden Ministerial, aunque no se consolida finalmente, la creación de la Unión para la Idoneidad Técnica Española (UITE), que busca ampliar el campo de actuación de la UEltc, al de la DPC. Sí se desarrolla sin embargo, una colaboración efectiva entre la Subdirección General de Normativa y Tecnología de la Edificación y el IETCC, en la participación en EOTA y en las actuaciones relacionadas con los DITE.

La CE publica las Guías para aplicación de la Directiva DPC 89/106/CEE: A. Designación Organismos Notificados, B. Control de Producción en Fábrica, C. Kits y Sistemas, etc.

En el IETcC, Vicente Más es sustituido por Antonio Blázquez, como Responsable y Coordinador del DIT y DITE.

1993: El año que Checoslovaquia después de 74 años de historia, se divide en República Checa y Eslovaquia. Primer atentado terrorista al World Trade Center de Nueva York: Mueren 6 personas. Bill Clinton es elegido Presidente de EEUU. (...)

Se modifica la DPC con la Directiva 93/68/ CEE. La EOTA aprueba sus Estatutos.
Alemania (El Instituto BAM), se retira de la UEAtc (pero volverá como DIBt). Los Institutos EMI de Hungría e ITB de Polonia, entran como observadores.

La UEAtc publica su declaración: "UEAtc 1993 and beyond", (La UEAtc y más allá) que reafirma la continuidad de la UEAtc frente a la EOTA.

M.C. Andrade es nombrada, de nuevo, directora del IETCC.

1994: El año que entra en vigor el Tratado de Libre Comercio de América del Norte, entre EEUU, Canadá y Méjico. Suecia, Austria y Finlandia se incorporan a Europa (ya son quince miembros). Descubrimiento de la partícula subatómica "alta quark". Elecciones en Sudáfrica que ponen fin al "apartheid". (...)

La UEAtc aprueba su Regla 04 relativa a las Confirmaciones del DIT entre sus miembros.

1995: El año que aparece el sistema operativo Windows 95. Se inaugura el Museo de Arte Moderno de San Francisco, diseñado por Mario Botta. Muere Lola Flores y también su hijo Antonio Flores. (...)

Se publica el RD 1398/1995, (sustituyendo al RD 1630/1992) en aplicación de la DPC 93/68/CEE, que modifica a su vez la DPC 89/106/CEE.

Se establece en la Orden de 1 de agosto, el Reglamento y las Normas de Régimen Interior de la Comisión Interministerial para los Productos de Construcción.

El Instituto húngaro EMI y el polaco ITB son aceptados como miembros de UEAtc (ya son quince miembros). El Instituto alemán DIBt está representado como invitado y el INCERC de Rumanía es aceptado como observador. Comienzan a publicarse los Informes Técnicos de la UEAtc (UEAtc Technical Reports). La última Guía se publicará en 2002.

La directora del IETcC, Carmen Andrade es elegida presidenta de la UEAtc. Lo será hasta 1999. Primera vez que un español/a ostenta la Presidencia de la Organización.

1996: El año que el superordenador Deep Blue de IBM vence por primera vez a Gari Kaspárov. El Partido Popular con José María Aznar, gana las elecciones generales españolas. Se detecta la enfermedad de las "vacas locas". Arturo Pérez-Reverte publica la primera entrega de la serie: Las aventuras del Capitán Alatriste. El arquitecto Rafael 
Moneo gana el premio Pritzker. El Club de fútbol Atlético de Madrid gana el "doblete": Liga y Copa del Rey. (...)

Se crea en París, la World Federation of Technical Assessment Organizations (WFTAO), con el IETcc como Organismo Cofundador. Esta Organización, única en el mundo, tiene como misiones principales, la promoción de las actividades relacionadas con el DIT y el desarrollo de procedimientos destinados a facilitar la confianza mutua en los DIT concedidos por sus miembros, entre los que se encuentran, además de la mayoría de los pertenecientes a la UEAtc: Australia, Brasil, Canadá, Israel, Japón, Nueva Zelanda, Rusia, SudÁfrica y EE.UU.

El Instituto rumano INCERC, cumple su segundo año como observador en la UEAtc. Será aceptado como miembro el año siguiente (1997). El Instituto FGW de Austria, se retira de la Organización, no habiéndose reincorporado hasta la fecha. El Instituto TZUS de la república Checa es aceptado como observador. El Instituto de Dinamarca ETA-DANMARK sustituye al SBI.

1998: El año que Juan Pablo II visita Cuba. Se funda la empresa GOOGLE. Hallan en Canadá el mayor excremento de dinosaurio del mundo: Podría ser un Tyrannosaurus. Sale el sistema operativo Windows 98. El Real Madrid gana frente al Milán su $7^{a}$ Copa de Europa. (...)

La UEAtc revisa sus Estatutos y Reglamento Interno y aprueba la Regla 05, relativa al procedimiento EUROAGRÉMENT, que permite obtener simultáneamente el DIT en varios países. Para ello, el Instituto que recibe la petición inicial, prepara un documento base (core document) al que se van añadiendo anexos nacionales en función de las diferentes exigencias de cada país. Es un procedimiento que se ha consolidado ya en la UEAtc, aunque sus expectativas eran mayores de las alcanzadas hasta el momento. Naturalmente tiene mucho que ver la coexistencia con el DITE europeo.

La revisión y aprobación de nuevos Estatutos y del Reglamento Interior de la UEAtc, genera una nueva Comisión denominada Comisión Técnica (Technical Commission) que se diferencia de la Comisión de Confirmación. Esta Comisión tratará los temas específicos que eran anteriormente objeto de las Comisiones Especializadas. También se aprueba un formato unificado para el Informe de Inspección de Fábrica (publicado en el Boletín n³7).

Se adopta la Directiva 98/34/CE que obliga a todos los Estados Miembros a comunicar a la CE todo proyecto (o modificación) de reglamentación técnica. La EOTA concede el primer DITE, al sistema HILTI de anclaje metálico para hormigón: ETA-98/0001.

La Comisión Técnica española del DIT aprueba el Reglamento para el Seguimiento de los DIT. Desde ese momento, todos los DIT concedidos por el IETcc son objeto de una "certificación permanente".

1999: El año que entra en vigor el euro. (En la UE sólo Dinamarca, Reino Unido y Suecia no han aceptado aún la moneda única). Un coleccionista anónimo paga en Nueva York 1.166 millones de pesetas por el cuadro Santa Rufina del pintor sevillano Velázquez. Primera gran condena a Philips Morris fabricante de los cigarrillos "Marlboro". La OPEP ratifica en Viena un nuevo recorte de producción de petróleo. La OTAN inicia el bombardeo de Yugoslavia. El último día del año fue el de la sicosis por la llegada del año 2000: "el efecto dos mil". (...)

El Instituto TZUS, de la República Checa y el Instituto DIBt, de Alemania son aceptados como nuevos miembros de la UEAtc. (Ya son 17 miembros).

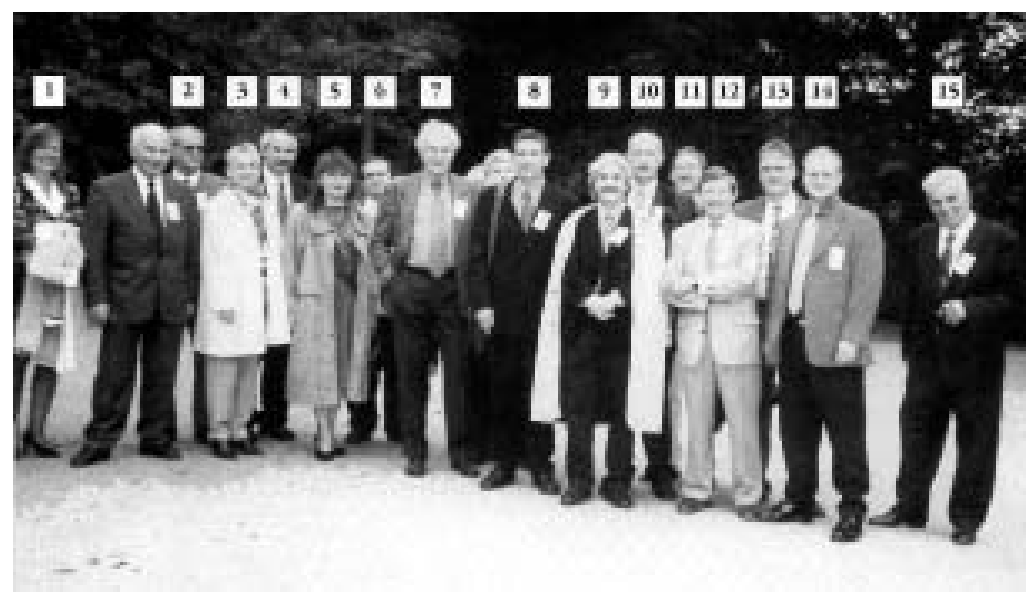

El Estado español publica la Ley 38/1999 de Ordenación en la Edificación (LOE). Lo que supone de facto que España elige como política de calidad, el SEGURO.

Primera notificación a la CE de designación IETcC como Organismo Autorizado de certificación (Approved Body 1219) para varias guías DITE. A partir de entonces, con cada aprobación definitiva de Guías se comunica por el Estado Español a la CE, los Organismos Notificados para cada producto específico.

Se crea en Bruselas el Grupo de Organismos Notificados de la DPC, tanto para productos tradicionales (cubiertos por Normas armonizadas) como innovadores (cubiertos por DITEs). AENOR representa a los Organismos españoles.

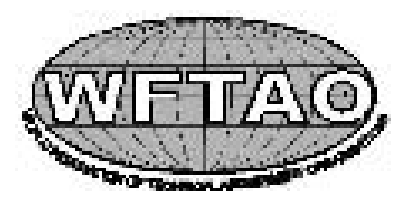

Logo WFTAO.

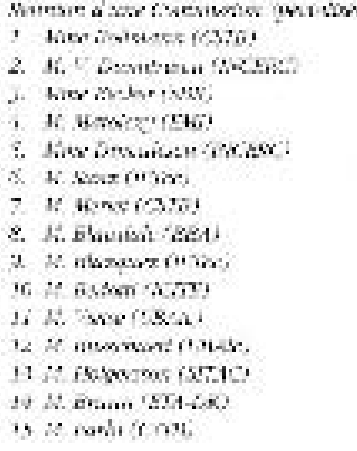

Reunión Comisión Especializada UEAtc. 


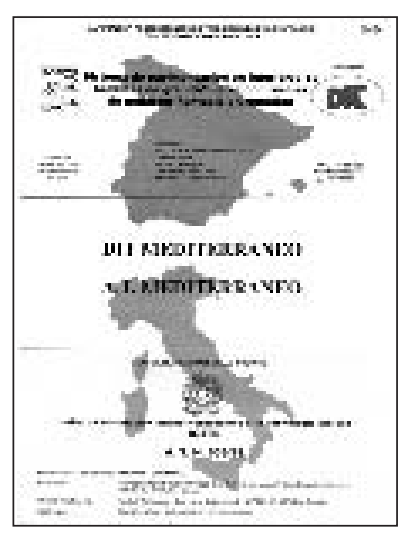

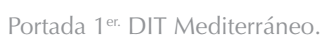

$40^{\circ}$ Aniversario UEAtc. Reunión en Madrid (2000)
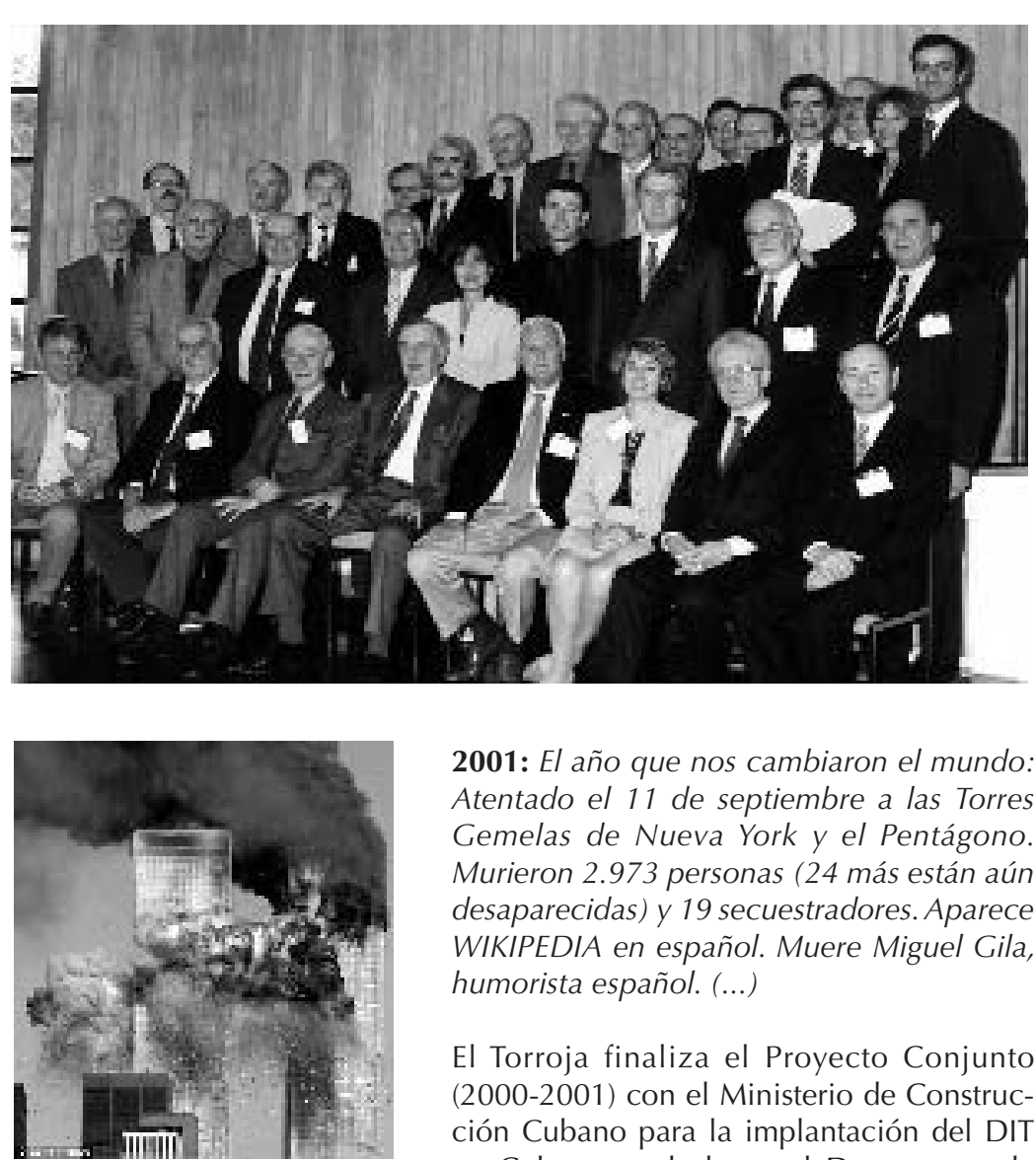

Torres Gemelas. Atentado 11-S-01.

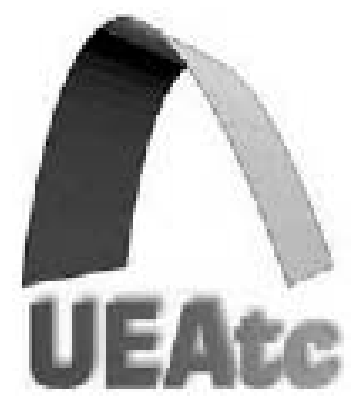

Nuevo Logo UEAtc.
El Torroja desarrolla un Reglamento Interno para la concesión del DIT Experimental (DITEX), basado en el ATEX francés expedido por el CSTB, que a diferencia de la implantación de este último en Francia, no logra asentarse en España.

El DITEX es aún una posibilidad para los fabricantes y técnicos que desean tener una evaluación rápida de una solución constructiva determinada para una obra específica.

El Torroja junto con el ICITE italiano, desarroIlan y conceden el primer DIT Mediterráneo, (DIT n ${ }^{\circ}$ 340/ATN 536/99), un documento resultado de la evaluación conjunta de ambos Centros, que se publica a la vez en las dos lenguas: español e italiano.

2001: El año que nos cambiaron el mundo: Atentado el 11 de septiembre a las Torres Gemelas de Nueva York y el Pentágono. Murieron 2.973 personas (24 más están aún desaparecidas) y 19 secuestradores. Aparece WIKIPEDIA en español. Muere Miguel Gila, humorista español. (...)

El Torroja finaliza el Proyecto Conjunto (2000-2001) con el Ministerio de Construcción Cubano para la implantación del DIT en Cuba, que da lugar al Documento de Idoneidad Técnica Cubano (DITEC), para el que ambos Organismos desarrollaron un Reglamento de Concesión basado en el DIT Español.

UEAtc cambia su logotipo.

2002: El año que se pone fin al curso legal de la peseta. Entra en vigor el Estatuto de Roma que crea el Tribunal Penal Internacional. Frente a las costas gallegas explota el tanque del petrolero "Prestige". "El Real Madrid gana su última (por el momento) Copa de Europa: la Novena. (...)
Se modifica la disposición adicional segunda de la LOE: "Obligatoriedad de las garantías por daños materiales ocasionados por vicios y defectos de la construcción", que no exigirá la garantía (referida en el apartado 1.c, del artículo 19) en el supuesto del autopromotor individual de una única vivienda unifamiliar para uso propio, una necesaria, aunque controvertida, modificación.

2003: Se desintegra al entrar en la atmósfera terrestre el trasbordador Columbia: Mueren sus siete tripulantes. Tropas de EEUU y otros tres países (Australia, Polonia y Dinamarca) invaden Iraq. Último vuelo del Concorde. Pérez Reverte toma posesión del sillón T de la RAE. (...)

El Torroja participa en el primer Euroagrément expedido por el BBA como promotor y el LNEC, UBAtc e IETCC como colaboradores. Ese año el Torroja concede un total de 37 DIT, de los cuáles 11 son renovaciones y 1 confirmación.

El IETcC, concede el primer Documento de Idoneidad Técnica Europeo (DITE) expedido en España: DITE 03/0054 al sistema Elastinor -Q.

UEAtc mantiene una activa participación en WFTAO, a través de sus miembros.

Juan Monjo es nombrado director del IETCC

2004: El año que nos cambiaron aún más el mundo: Atentado terrorista el 11 de marzo en Madrid: Murieron 190 personas (ningún terrorista). En la UE se integran: Chipre, República Checa, Eslovaquia, Estonia, Hungría, Letonia, Malta y Polonia. (...)

El día de los atentados, la UEAtc celebraba su reunión anual de Coordinación en Helsinki.

UEAtc edita su, hasta ahora, último "Technical Report" para: "Sistemas de láminas discontinuas bajo teja". El trabajo continuará sin embargo, con la redacción de Guías horizontales: Sostenibilidad, reciclado, vida útil, etc.

2005: El año que entra en vigor el protocolo de Kioto. Muere el Papa Juan Pablo II. Los españoles aprueban en referéndum la Constitución Europea, que después, no será aprobada en otros Países miembros. Nadal gana su primer Roland Garros (ganará cuatro consecutivos). Madrid no obtiene la designación de ciudad olímpica para el 2012, que se concede a Londres. Fernando Alonso gana su primer campeonato del mundo de Fórmula 1 (ganará dos consecutivos). (...) 
Se publica el RD 312/2005 sobre clasificación de productos frente a la reacción y resistencia al fuego, que será modificado el 2008.

La UEAtc desarrolla el procedimiento denominado "Application document" o "Document d'application", un nuevo documento que complementa al marcado $C E$, ya sea basado en Norma Armonizada o DITE, considerando, entre otros aspectos, la evaluación de la puesta en obra y un nivel superior del control de producción en fábrica.

El Torroja desarrolla este documento en su Procedimiento IETcc-0405-DP, Ilamándolo: DIT plus. Este mismo año se concederá ya el primer documento español. El DIT plus 469-p al Mortero Monocapa CLR 12.

2006: El año que entra en vigor la Ley antitabaco. Se inaugura la terminal T4 del aeropuerto de Barajas. Fidel Castro delega por enfermedad todas sus funciones a su hermano Raúl. La selección nacional de baloncesto española se proclama por primera vez campeona del mundo. Muere en Chile el dictador Augusto Pinochet. (...)

Este año se construyeron casi novecientas mil viviendas en España, récord difícilmente igualable en el futuro.

Se publica el Real Decreto 314/2006 por el que se aprueba el Código Técnico de la Edificación, cuyo "enfoque prestacional", supuso un avance sin precedentes en la regulación española del sector de la construcción. En su Parte I, artículo 5.2.5, se indica:

"Se considerarán conformes con el CTE los productos, equipos y sistemas innovadores que demuestren el cumplimiento de las Exigencias Básicas del CTE referentes a los elementos constructivos en los que intervienen, mediante una evaluación técnica favorable de su idoneidad para el uso previsto, concedida, a la entrada en vigor del CTE, por las entidades autorizadas para ello por las administraciones públicas competentes en aplicación de los criterios siguientes: (...)

Se publica el RD 315/2006 por el que se crea el Consejo para la Sostenibilidad, Innovación y Calidad de la Edificación.

2007: El año en el que la crisis económica ("recession", en inglés) comenzó a "preocupar" a los españoles. Se firma el acuerdo de Schengen para armonizar los controles fronterizos. El "Grupo Intergubernamental de Expertos sobre Cambio Climático" (IPCC) entrega en Paris su Informe sobre el cambio climático. Nicolás Sarkozy asume la presidencia de Francia. Un melón de 2.100

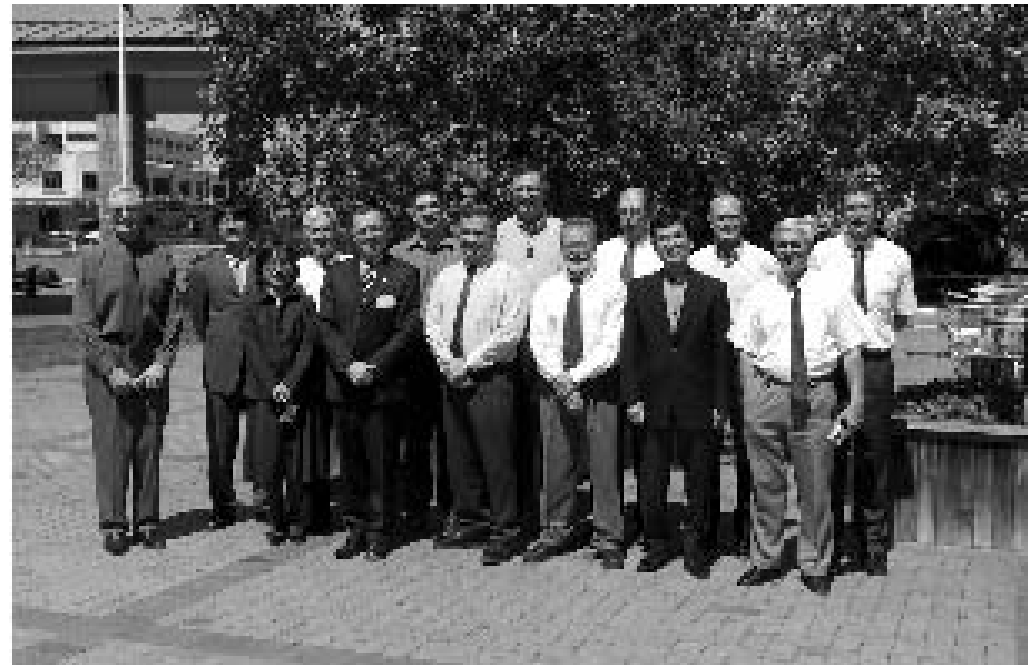

años es descubierto por los arqueólogos en Japón. Apple Inc., presenta el nuevo iphone. Rumanía y Bulgaria entran en la UE. Muere el Fary, cantante español. (...)

Se publican el RD 1371/2007 de aprobación del DB-HR Protección contra el ruido, y el RD 47/2007 para el Procedimiento básico para la certificación energética de edificios de nueva construcción.

El Instituto de Ucrania NIISK es aceptado como observador de la UEAtc, durante el período establecido de dos años (será aceptado en el 2009 y los Miembros serán en total: 18).

Este año se alcanza el número más alto de DIT concedidos por el IETcC: 48.

El Director del IETcc, Juan Monjo, es elegido presidente de la UEAtc. Lo será hasta el 2008.

2008: El año que la crisis económica se manifestó en toda su crudeza. Se registra la mayor caída del Ibex 35 (cae hasta los 12.265 puntos). El AVE llega a Barcelona. Exposición internacional en Zaragoza bajo el lema: "Agua y desarrollo sostenible". Barack Obama es elegido Presidente de EEUU. Wikipedia cifra la población mundial en 6.706.993.152 de personas. (...)

Se publican el RD 110/2008 sobre clasificación de productos frente a la reacción y resistencia al fuego, que modifica el RD 312/2005 y la Orden VIV/1744/2008 por la que se regula el Registro General de CTE.

Se hace formal la Propuesta (en fase por tanto de aprobación) 2008/0098 del Consejo de la Unión Europea de Reglamento del Parlamento Europeo y del Consejo por el que se establecen condiciones armonizadas para la comercialización de los productos
Reunión de WFTAO en Nueva Zelanda (2003). Participaron los Miembros de UEAtc de: Reino Unido, Hungria, Dinamarca, República Checa y España.

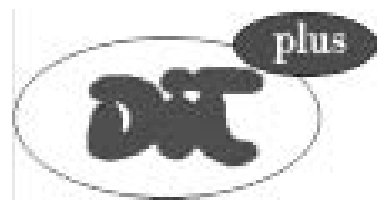

Logo DIT plus

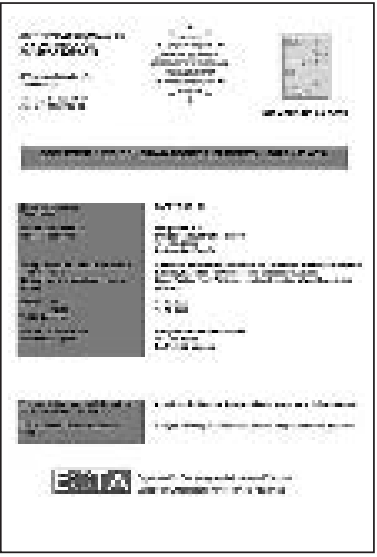

Portada $1^{\text {er. DITE concedido por }}$ el IETcc. 
de construcción. (Reglamento que sustituirá a la DPC).

El IETcc concedió a final del 2008 el DIT $\mathrm{n}^{\circ}$ 523-p/08. Desde 1962, suponen 552 documentos (incluyendo 76 Confirmaciones y 54 DIT plus).

También se alcanzaron los 51 DITEs, desde el primero concedido en el 2003.

El IETcc, como cada año, presenta su Informe/Memoria anual a la UEAtc en la que recoge, siguiendo un esquema de contenido, previamente acordado en la UEAtc, los aspectos más significativos ocurridos en el año, relacionados con el DIT y la evaluación de la innovación. Informe que se discute y valora en la reunión anual de la Comisión de Coordinación, junto con los presentados por el resto de Institutos Miembros.

Dicho Informe, se resume en las siguientes páginas:

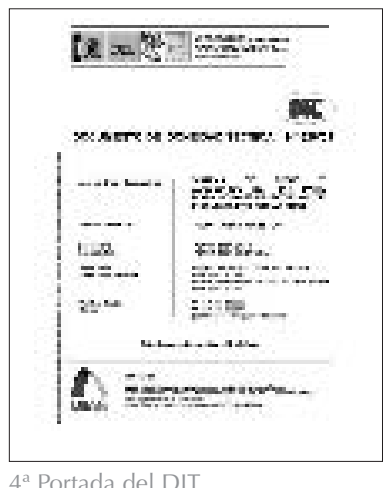

$4^{\text {a Portada del DIT. }}$

\section{Resumen de la Memoria presentada a la Comisión de Coordinación n ${ }^{\circ} 55$ de la UEAtc}

\section{SUMMARY}

During the last semester 2008 the Spanish economy had a negative evolution.

Comparing with to 2007 the number of National Approvals (DIT, DIT plus, etc.), issued by the IETCC has decreased; although the general evolution (We think it should be considered biannual periods for comparisons) maintains the increasing process together with the number of European Technical Approvals issued in the field EOTA. Anyhow for innovative products not covered by ETA Guidelines, National Approvals still remains as the first option for Spanish manufacturers, due to the fact that all the certificates issued by IETCC (DIT and DIT plus) including those based on UEAtc's Confirmation Procedure contain the necessary information to justify the fulfilment of the Spanish Building Code Regulations (CTE).

The number of agréments issued in 2008 as well as the number of applications underway confirms the interest and confidence of the Spanish manufacturers on this procedure. The effects of the recession and the consequences on the number of applications for DIT, DIT plus and ETAs, should be evaluated in the following months.

\section{AGRÉMENTS ISSUED}

\subsection{Number of new Agrément Certificates issued and the subjects covered}

The number of DIT granted during 2008 has been 22 with 4 DIT plus (several documents were ready to be endorsed at the very beginning of 2009). The subjects covered and the ratio is shown in the following figure:

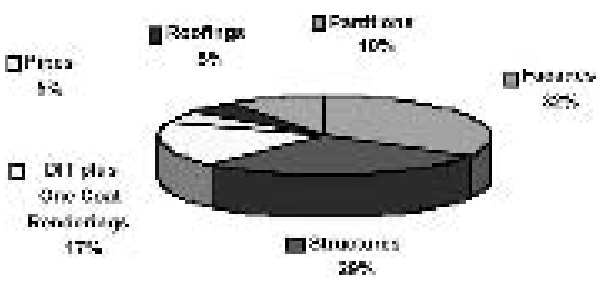

During 2008 the number of TA issued for structural and cladding systems have increased while for "one coat mortar" (DIT plus) the number has decreased.

The Comparison between DITs (UEAtC) and DITEs (EOTA), issued yearly by IETCC is shown in the following figure:

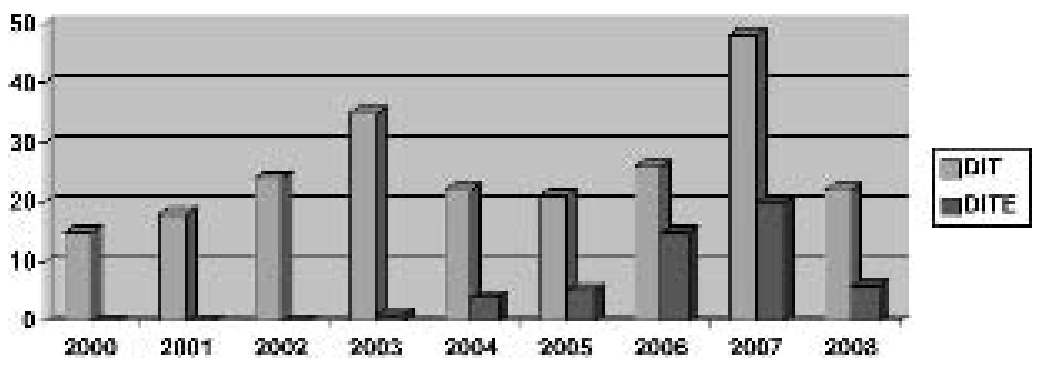

\subsubsection{Number of current valid DIT} (Agrément):

The number of current valid Agrément under

Permanent Surveillance is: 170 


\subsection{Number of Confirmation Certificates in 2008}

2.2.1 Number of Confirmation Certificates issued: 0 . Nevertheless a Spanish DIT plus: Sistemas de revestimiento de fachadas ventiladas VMZ Panel de fachada $N^{\circ}$ 519p/08 (Cladding kits) has been issued taken into account the information provided in the Constat de Tradicionalité $n^{\circ}$ 2/04/1067 issued by CSTB

2.2.2. Number of Confirmation Certificates underway.: 10

2.2.3 Number of Euroagrément issued: $\mathbf{0}$

2.2.4 Number of Euroagrément underway: o

\subsection{Other documents issued e.g. assessment/test reports}

DIT's Unit is also working in several research projects and Technical Reports for different topics like construction pathology studies in buildings. A book titled "Marcado CE para productos de construcción. Código Técnico y marcas voluntarias" (CE marking for construction products, National Technical Building Code and Voluntary Marks), ISBN 84-931709-7-6, written by Antonio Blázquez \& Luis Vega, was published and disseminated to manufacturers and other agents of the sector.

This book provides information about Spanish's situation in relation with innovative products as well as the different UEAtc's procedures and services offered by UEAtC's members.

\section{NEW PRODUCT AREAS}

\subsection{New subjects areas}

During 2008 a number of DIT's applications were received for:

- "Fire protective mortars"

- "New Solar collectors based in thermodynamic systems"

- "New solar panels as a part of cladding kits systems"

- "Fire stops devices for pipes"

- "Prefabricated buildings units"

- "Technical floors"

And DIT plus for the next subjects:

- "Underlay for discontinuous roofing". (CE marking based on hEN)

- "Underlay for walls". (CE marking based on hEN)

- "Flexible sheet for waterproofing-reinforced bitumen sheet for waterproofing of concrete bridge decks another concrete surface trafficable by vehicles" (CE marking based on $h E N$ )

- "Insulation mortars for rendering" (CE marking based on hEN)

- "TPO membranes" (CE marking based on $h E N)$.

- "External Thermal Insulation Composite Systems" (CE Marking based on ETAG)

- "Liquid applied roof waterproofing kits" (CE Marking based on ETAG)

\subsection{Developments in other areas}

In addition to working on DIT and ETA certification managed at the DIT's Unit, IETCC has conducted construction product research in the following fields:

- Structural failure and risk analysis.

- Structural analysis-theory.

- Experimental analysis of structures.

-Structural use of new composite materials (FRP).

-Building and construction component systems: Industrialization, new buildings, new distribution and finish systems.

- Vitreous and ceramic building products.

-Construction process rationalization and sustainability.

- Comfort in indoor environments: lightning, noise, vibration, quality of indoor air and so on.

- High efficiency energy systems.

-Constructions solutions using alternative, low pollution energy.

-Climatic modelling to study construction material and system behaviour.

- Performance of lightweight facades.

- Studies of harmful natural radiation. Radon in building construction.

- Study on pressurized and non-pressurized water piping.

- Study on mechanical action in underground plastic piping and ducting.

- Characterization of drainage system components.

-Renforcement corrosion: electrochemical techniques, corrosion mechanisms, corrosion prevention.

-Concrete durability: aggressive agent transport and test methods, characterization of aggressive environments (for concrete), effects of fire.

-Structural repair: traditional methods, advanced methods (chloride removal), Realkylinization.

-Environmental: secondary materials in cement.

- Heritage conservation: research on historical materials and mortars.

- Portland cements and new binders.

-Industrial waste and by-product re-use. Waste containment. Depollution and environmental analysis.

- Historic heritage. 


\subsection{Developments intended for the future}

3.3.1. Activities for national promotion of Technical Approval

Disseminating information on the DIT procedure is one of the IETCC's priority tasks every year.

With this aim in mind, congresses, seminars and workshops were attended and presentations made both in Madrid and the rest of Spain in 2008.

At the international level the IETCC maintains working relations with South American countries, as well as participates actively in other organisations, such as: EOTA, WFTAO, ENBRI, CIB, RILEM, FIB, ISO, CEN, ALCONPAT and CONPAT Conference.

\subsubsection{Activities for products}

DIT's content (also for DIT's confirmations) has been revised to include justifications (for each essential requirement) about the Spanish Building Code (CTE).

New group agreements with associations or groups of product manufacturers were concluded to co-ordinate collective DIT procedures. The joint process has proved to be highly effective in preventing market distortion, reducing overall DIT costs and ensuring equitable conditions with the simultaneous issue of DIT certification. This experience has been taken also for ETA applications.

\subsection{Number of applications in progress}

The number of applications under way are 65 for DIT (10 Confirmation's applications) and 30 for DIT plus.

\section{ANY PROBLEMS EXPERIENCED}

There are some problems expected to be mentioned.

From one side the recession of the sector that seems to be more important than any other previous crisis and with some Spanish particularities due to the burble effect in the construction sector of the previous years. From other side the difficulties of manufacturers and other agents to understand the foreseen changes for the future: National Technical Approvals, CE marking, CPD revision, etc.

REf. IETCC/AB/UEAtc/2008 